\title{
CRITERIO DE OPTIMIZACION POR COMPENSACION EN EL DISEÑO DE BOTELLAS CON SUPĖRICIES LATERALES DE REVOLUCION USADOS POR EMPRESAS EN LA COMERCIALIZACION DE ALIMENTOS Y BEBIDAS
}

\section{OPTIMIZATION CRITERION BY COMPENSATION FOR DESIGNING BOTTLES WITH SURFACE OF REVOLUTION USED BY FACTORIES FOR COMMERCIALIZING FOODS AND DRINKS}

\author{
Lizandro B. Reyna-Zegarra $^{a}$, Teodoro Moore-Flores ${ }^{b}$
}

\section{RESUMEN}

En el presente artículo, se expone el resultado de un trabajo de investigación realizado con el propósito de validar un criterio a seguir con la finalidad de optimizar el ahorro de material en el diseño de botellas comerciales con superficies laterales de revolución y bases planas fundamentalmente. A tal criterio de optimización se le denomino Criterio de Optimización por Compensación el cual usa como herramienta principal los métodos numéricos y un programa computacional. La característica fundamental de diseño optimo es que el contenedor debe ser talque su área superficial interna debe ser mínima para un cierto volumen dado. El criterio quedo validado al mostrarse que en casi la totalidad de botellas comerciales se usa mas material que el establecido por el Criterio de Optimización por Compensación.

Palabras clave: Optimización, Diseño de botellas comerciales.

\begin{abstract}
In the present article is expounded the result of a research work performed in order to validate a criterion to fallow to optimize material for designing commercial bottles with lateral revolution surfaces and flat bases mainly. Such optimization criterion was called Optimization Criterion by Compensation which uses as main tools, numerical methods and a computer program. The fundamental characteristic of optimum design is that the container must be in such a way the area of its total internal surface must be a minimum for a given volume. Such criterion was validated when it was shown that in most commercial bottles it is used much more material than the Optimization Criterion by Compensation tell us.
\end{abstract}

Key words: Optimization, Design of commercial bottles.

\section{INTRODUCCION}

Durante las etapas de producción de un articulo, hasta finalmente ser enviado al publico, los fabricantes y comerciantes deben enfrentarse cada día a un mercado y una sociedad mas exigente, en donde el envase tiene que satisfacer no solo la necesidad de contener, proteger, preservar, comercializar y distribuir mercancías, sino también los alcances de su disposición posterior a su uso principal, la reutilización y reciclaje de los materiales, impactos ecológicos así como costos. Por esto, se crea la necesidad de diseñar envases adecuados, que hace obvia la necesidad de generar y transmitir los conocimientos de la ciencia y tecnología, en donde el concepto de optimización resulta ser de gran importancia.

Puesto que la mayoría de envases cuyas formas quedan determinadas por superficies de revolución son fabricados de material de vidrio o plástico, las empresas que decidan aplicar criterios de optimización en su fabricación, podrán alcanzar logros tales como:

1. Disminución de impacto ambiental: Al reducir el área superficial de los envases manteniendo el volumen constante, se estará reduciendo el material requerido, con lo cual menor será la necesidad de la producción de plástico, principal responsable de la contaminación ambiental.

2. Reducción de desechos: Parte de la problemática ambiental radica en la acumulación excesiva de desechos entre los cuales se encuentra fundamentalmente los envases plásticos. Al optimizar, los envases salen al mercado con menos material y por lo tanto se reduce la cantidad de desechos antes de que llegue al consumidor.

3. Rebaja de costos por concepto de materia prima: Las empresas productoras de envases al requerir menos material por unidad de envase para su fabricación requerirán de menor capital para operar.

4. Ahorro energético: Al requerirse menor cantidad de materia prima para la fabricación de envases plásticos y de vidrio, menor cantidad de energía se requerirá para la obtención de tal materia prima. Menor cantidad de energía se requerirá también para su transporte a centros de envase y comercialización debido a la disminución de peso por envase.

$a, b$ Departamento de Matemática de la Universidad Nacional de la Santa, Chimbote, Perú 
5. Beneficiosa imagen ambiental: Un efecto de la conciencia ambiental colectiva el cual debe fomentarse, es que las personas desean contribuir a conservar el medio ambiente, pero que se sienten impotentes ante las necesidades de consumo y al no conocer una alternativa que no demande sacrificios mayores, deja esa preocupación por el ambiente a un segundo plano. Con la propuesta de optimización, se le da al consumidor la oportunidad de elegir productos en envases que traen incorporado este ahorro que ellos quieren lograr sin mayor esfuerzo que el de preferir estos envases optimizados, que a los que no presentan un diseño optimo. Además, el hecho de que un producto no contamina más de la cuenta, es un factor importante en publicidad, debido a que resulta atractivo y sobresaliente gracias a la tendencia que deben tener los consumidores a elegir productos amigables con el medio ambiente.

6. Disminución de los precios de los productos de consumo: Al disminuir el costo de fabricación de cada envase por requerir menos material, el producto a comercializarse en el envase disminuirá en su precio, pues el precio del envase lo paga el consumidor y este precio será menor.

\section{CRITERIO DE OPTIMIZACION DE ENVASES DE FORMA CILINDRICA}

La investigación sobre los niveles de optimización de envases comerciales de forma cilíndrica mostró que de una muestra de 30 envases tomadas al azar, el $88.9 \%$ de envases que usan las empresas peruanas no siguen criterios de optimización en su diseño, mientras que el $75 \%$ de envases de empresas extranjeras si lo hacen.

Diseñar un envase de forma cilíndrica recta y de capacidad volumétrica usando la menor cantidad de material posible, conlleva ha determinar un mínimo para la función

$$
A(r)=2 \pi r^{2}+\frac{2 V}{r}
$$

Donde $V, A, r$ son el volumen, área total y radio del cilindro respectivamente.

Se encuentra que al determinar los extremos de (1), el numero critico que viene a ser el radio correspondiente al punto de mínimo, está dado por la formula

$$
r=\sqrt[3]{\frac{V}{2 \pi}}
$$

la cual permite determinar la altura igual a

$$
h=\sqrt[3]{\frac{4 V}{\pi}}
$$

Luego el área mínima en función del volumen estará dada por

$$
A(V)=\left(\frac{6 \pi}{\sqrt[3]{4 \pi^{2}}}\right) \sqrt[3]{V^{2}}
$$

Cuanto más grande es el volumen del envase, mayor oportunidad se tiene en disminuir el radio con el fin de aumentar la altura del envase a cuenta de que el área no se aleje mucho del mínimo. Ver figura 1.

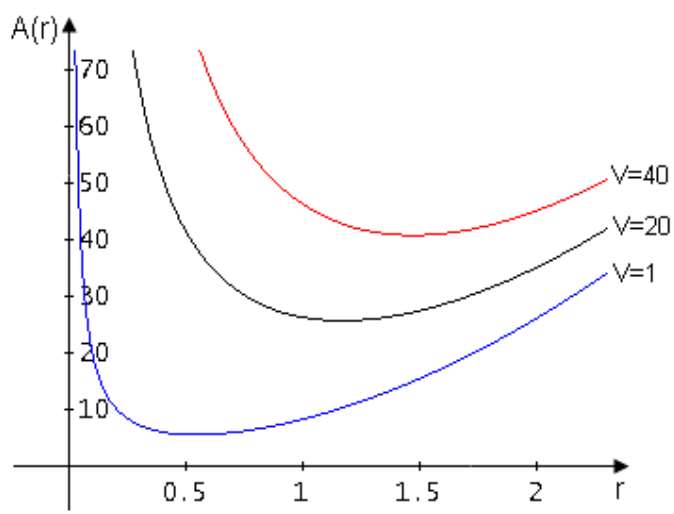

Figura 1 Grafica del área total $A$ de un cilindro en función del radio $r, A(r)=2 \pi r^{2}+\frac{2 V}{r}$. Con fines comerciales siempre se busca aumentar la altura del envase. Cuando el volumen es grande, podemos desplazarnos a la izquierda del mínimo variando el radio sin que se aleje demasiado del área mínima.

\section{CRITERIO DE OPTIMIZACION POR COMPENSACION}

En el caso del diseño de envases con superficies laterales de revolución cuya silueta resulta ser caprichosa debido a que es impuesta por la estética y otras consideraciones, no es posible establecer una ecuación de tal manera que se pueda seguir los mismos pasos que para el caso del diseño de envases de forma cilíndrica recta. En este contexto, surge la siguiente interrogante [2]:

¿Cuál es el criterio que debería seguirse con el fin de optimizar el diseño de botellas comerciales con superficies laterales de revolución de tal manera que se use la menor cantidad de material posible en su fabricación?

Como respuesta al problema planteado, se formulo la siguiente hipótesis[2]:

Un criterio que nos permitirá optimizar el diseño de botellas comerciales con superficies laterales de revolución de tal manera que se use la menor cantidad de material posible en su fabricación, es el Criterio de Optimización por Compensación.

Se entiende por Criterio de Optimización por Compensación[2], aquel que consiste primero en diseñar óptimamente un cilindro cerrado recto y luego redistribuir el total del volumen y área que se retire de este con el fin de dar la forma deseada a una botella que se desea fabricar, dando como resultado una botella cuya superficie lateral corresponde al de una superficie 
de revolución y cuyas bases son planas. El volumen del envase resultante se debe mantener igual al del cilindro recto. Matemáticamente, el criterio de optimización consiste primero en determinar el área mínima que debe tener un cilindro recto para un volumen fijo dado. Luego a partir de esto, usando un programa de computadora elaborado para el propósito, manteniendo el volumen constante deformar el cilindro preservando la isometría ${ }^{1}$ en el proceso hasta llegar a la forma del envase deseado. Cilindro y botella final deben resultar ser isométricos. Puede ocurrir que la isometría se rompa talque el área superficial interna de la botella sea menor que la del cilindro de donde se partió [4].

En el esquema del Criterio de Optimización por Compensación para optimizar (minimizar) el área de envases manteniendo el volumen constante, se procede como sigue:

\subsection{Conocer la capacidad volumétrica del envase} a optimizar.

2.2 Optimizar el área de un cilindro cerrado recto cuya capacidad volumétrica sea igual a .

2.3 Seleccionar alguna función conocida o tabular para obtener un conjunto de puntos que por interpolación numérica se pueda determinar la función que describa la curva deseada que pasa por dichos puntos con el fin de determinar las formas en las zonas deseadas del envase cilíndrico optimizado de 2.2 .

2.4 En papel milimetrado graficar un suficiente numero de puntos previamente tabulados en 2.3 que describan la forma deseada en las zonas del envase cilíndrico de 2.2

2.5 Valiéndose de un programa de computadora interpolar los puntos considerados en el paso $2.4 \mathrm{y}$ calcular el área de la superficie de revolución que genera la curva al girar alrededor de un eje fijo así como el correspondiente volumen del sólido de revolución, correspondientes a las zonas donde se va ha dar la forma al envase.

2.6 En cada zona a dar forma al envase, se deberá remover un sólido con superficie externa de forma cilíndrica y superficie interna de forma determinada por la superficie de revolución que da la forma al envase. Calcular la diferencia de áreas entre la superficie cilíndrica y la superficie de revolución del sólido removido. Con esta diferencia se deberá formar un cilindro sin bases cuyo volumen sea aproximadamente igual al perdido al remover el sólido en la zona del cilindro optimizado con el fin de obtener la forma deseada en dicha zona.

2.7 Acoplar el cilindro sin bases obtenido en el paso 2.6 en la zona cilíndrica del cilindro optimizado. Así se ha

${ }^{1}$ En este contexto, debemos entender por isometría a la preservación de las áreas correspondientes a la del cilindro punto de partida y de la botella punto de llegada durante el proceso de deformación. obtenido un envase optimizado con superficie lateral de revolución y bases planas fundamentalmente. Ver figura 2.

La dificultad que se presenta en el paso 2.6, es como determinar la curva que dará la forma al envase, de tal manera que al remover el sólido a que se refiere, de la diferencia de áreas entre la de la superficie cilíndrica externa y la de la superficie de revolución interna se pueda construir un cilindro sin bases con volumen igual al volumen del sólido removido. Para ello se deberá valer del método del tanteo en la determinación de las curvas hasta alcanzar una aproximación adecuada.

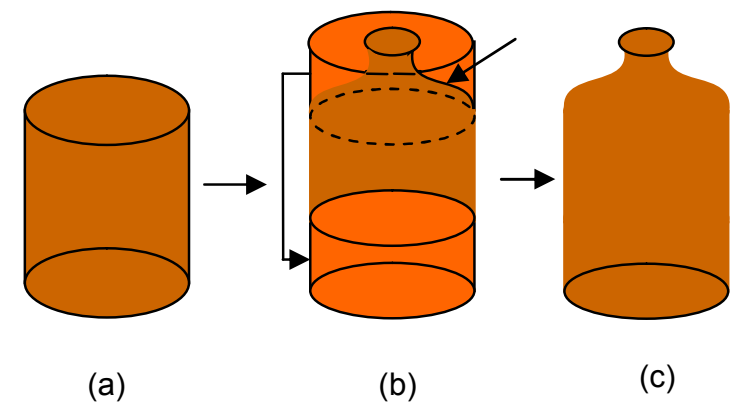

Figura 2 Secuencia esquemática en el diseño de una botella comercial siguiendo el Criterio de Optimización por Compensación. (a) Cilindro de volumen $V$ y área mínima $A$, a partir del cual se diseñara la botella deseada. (b) La flecha superior de la derecha, señala la generatriz de una superficie de revolución, limite de dos sólidos, una interna similar a una botella y otra externa cuya superficie pertenece al cilindro. El sólido externo con límite interno la superficie de revolución, es removido y redistribuido bajo una forma cilíndrica en la parte inferior del cilindro (como muestra la flecha del lado izquierdo) sin alterar ni el área superficial ni el volumen. (c) Botella obtenida y que corresponde a un sólido de revolución cuya área superficial total es igual a la del cilindro de área mínima.

El objetivo es pues establecer como criterio valido, al Criterio de Optimización por Compensación, el cual permita diseñar botellas con superficies laterales de revolución usando la menor cantidad de material posible en su fabricación respecto a la cantidad de material que se viene usando para fabricar las botellas comerciales de hoy.

Con el fin de verificar la validez de la hipótesis se seleccionaron botellas de marca prestigiosa y se determino si lo que el Criterio de Optimización por Compensación predice en lo que respecta a la cantidad de material necesario para la construcción de una botella es igual o menor que la cantidad de material usado en la construcción de las botellas seleccionadas. Llevado a cabo el análisis de las botellas comerciales vigentes [3], se encontró que de una muestra de 18 botellas tomadas al azar, estas tienen un error en promedio de $19 \%$ respecto del óptimo establecido por el Criterio de Optimización por Compensación. En el caso de botellas individuales se registró botellas cuyos diseños eran tal que en su fabricación requerían hasta de un $52.2 \%$ mas de material que el establecido por el Criterio de Optimización por Compensación.

Es importante indicar que al hacer el análisis de los envases indicados, al rediseñarlos con el fin de 
optimizar, todos los criterios que se siguieron en la fabricación del envase original se mantienen en el nuevo envase rediseñado a excepción de el hecho que la altura del envase disminuye y se ensancha lateralmente. Fundamentalmente se preserva el espesor de la pared del envase original.

Para realizar el estudio se preciso de herramientas matemáticas necesarias tales como Métodos Numéricos y uso de software Matlab 7.2 con el fin de elaborar un programa para llevar a cabo la deformación preservando la isometría y manteniendo el volumen constante. Igualmente se tuvo la necesidad de contar con un laboratorio básico para realizar el análisis de los envases tomados como muestra y así poder contrastar la hipótesis.

Es merito del autor el haber ideado, formulado, establecido el método y técnica así como la denominación de Criterio de Optimización por Compensación para diseñar botellas con superficies laterales de revolución minimizando el uso de material en su fabricación.

\section{APLICACIÓN DEL CRITERIO DE OPTIMIZACION POR COMPENSACION}

Como aplicación de lo expuesto, en la figura 3 se muestran a escala tanto el envase original como el envase optimizada en extremo, para el caso de una botella de gaseosa Fanta de capacidad $500 \mathrm{ml}$, producto de la Coca Cola.

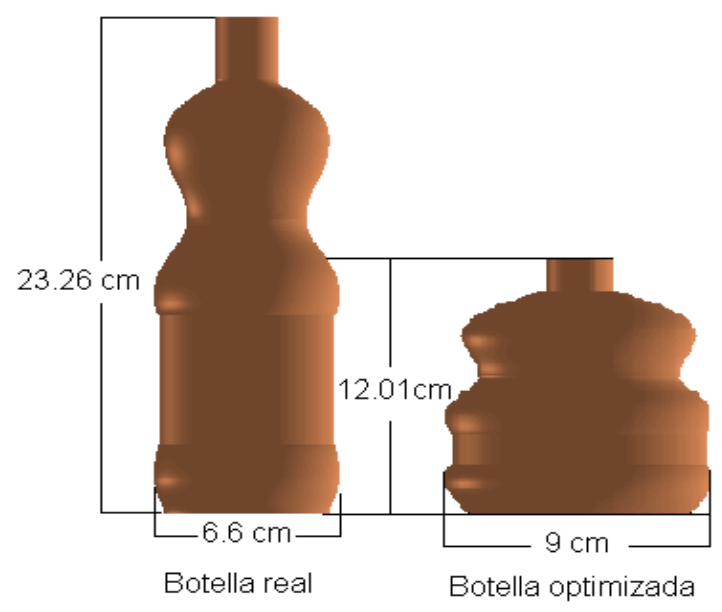

Figura 3 Al optimizar al extremo, la nueva botella obtenida resulta tener una altura igual a casi la mitad de la original con un correspondiente ensanchamiento como se aprecia en los datos numéricos de la figura.

Como resultado de la contrastación de la hipótesis realizada, el Criterio de Optimización por Compensación quedo validado[2,3]. En el caso del envase de gaseosa Fanta que se muestra en la figura 3, al aplicarse el Criterio de Optimización por Compensación en el rediseño del envase conservando su volumen, espesor de la pared del envase y manteniendo una forma muy parecida todo respecto del envase original, quedo establecido que se puede ahorrar $74.442 \mathrm{~cm} 2$ de material que traducido en gramos equivale a un ahorro de 4.18gr por envase, representando un error del $20.7 \%$ respecto del optimo. Lo que fundamentalmente varia en el rediseño es la altura de la botella, disminuyendo de $23.26 \mathrm{~cm}$ a $12.01 \mathrm{~cm}$, además de un correspondiente ensanchamiento lateral de la botella de $6.6 \mathrm{~cm}$ a $9 \mathrm{~cm}$.

Se debe indicar que no es posible evitar la reducción de la altura del envase salvo a costa de aumentar el uso de material, sin embargo, no necesariamente se tendrá que diseñar un envase en extremo optimo, permitiendo el método controlar el nivel de optimización de acuerdo a la conveniencia deseada.

El caso de los envases farmacéuticos merece especial atención, pues casi la totalidad de estos está lejos estar optimizados. Se sostiene que los envases en los que se comercializan bebidas gaseosas, néctares, jugos, yogures, etc. deben ser lo mas grandes posibles en altura a fin de lograr que lo que contienen se venda mas debido al impacto que tendrán ante le consumidor, sin embargo, ¿debería aplicarse este mismo criterio a los envases farmacéuticos?, ¿Qué importa mas en un envase farmacéutico? Lo que contiene, o el tamaño y estética del envase. Un buen envase farmacéutico debería promocionar la economía en su fabricación con el fin de reducir la contaminación que pueda causar.

No es difícil ver que si por cada envase se ahorra un gramo de resina plástica, para una producción de un millón de envases, en total se habrá ahorrado una tonelada de plástico. En el caso de la botella de gaseosa Fanta se puede ahorrar alrededor de 4 gramos por envase, por lo tanto se ahorraría 4 toneladas de esta resina.

No es necesario hacer una investigación exhaustiva para darse cuenta que las formas y tamaños de las botellas están de alguna manera estandarizados a nivel mundial. Luego, estas no se ajustan al Criterio de Optimización por Compensación. La pregunta es ¿Cuántos millones de envases se producen en el mundo y cuantos miles o millones de toneladas de resina se podría evitar que vengan al mundo si se decidiera optimizar aun mas el uso de resinas plásticas en la fabricación de botellas? Se afirma que el reciclaje es la solución a la contaminación por residuos plásticos, ¿Cuántas veces se puede reciclar dichos residuos, antes que se produzca "desgaste"? Aun así, cualquier proceso de reutilización o transformación involucrara gasto de energía, uso de sustancias químicas contaminantes, etc.

Una buena alternativa a la solución de la contaminación ambiental podría muy bien empezar por la concertación entre las empresas que usan botellas para comercializar sus productos, al tomar la decisión de optimizar aun más el diseño de sus envases, y por que no, una optimización al extremo. La búsqueda de desterrar la preferencia del consumidor por productos envasados en botellas de mayor altura frente a los mismos envasados en botellas de menor altura pero que contienen el mismo producto de la misma calidad y cantidad debe ser tarea de los departamentos de marketing del las empresas y en general de todos: colegios, universidades, demás instituciones y sociedad en general. 
Se concluye que el Criterio de Optimización por Compensación es un criterio valido, pues permite minimizar aun más material del lo que se viene usando en la fabricación de envases comerciales. Al preservar además el espesor de la pared del envase original en el caso de rediseño, el nuevo envase ha de resultar más sólido y macizo, con mejor equilibrio y ocupara menor volumen debido a la reducción de material requerido para su fabricación.
Los autores expresan sus más sinceros agradecimientos a los estudiantes López Rivera, S., Lavandera Alva, A., Cerna Correa, J. y demás compañeros, todos estudiantes de Ing. Agroindustrial de la Universidad Nacional del Santa por su valiosa colaboración durante el desarrollo del método de optimización a que se refiere el presente artículo.

\section{REFERENCIAS BIBLIOGRAFICAS}

[1]. Reyna L. Llanos R. et al. Niveles de aplicación de los criterios de optimización en la fabricación de envases de forma cilíndrica que utilizan las empresas productoras de alimentos y bebidas. Proyecto de investigación $2005-$ UNS

[2]. Reyna L. Moore T. et al. Criterio de Optimización por Compensación en la fabricación de envases con superficies laterales de revolución y bases planas. Proyecto de investigación 2006 - UNS

[3]. Reyna L. Cedron E. et al. Niveles de aplicación de los criterios de optimización en el diseño geométrico de envases comerciales con superficies laterales de revolución en base al Criterio de Optimización por Compensación. Proyecto de investigación 2007 - UNS

[4]. Pak I. Inflating the cube without stretching. Department of Mathematics, M.I.T. Cambridge, 2006.

[5]. Sherman K. Stein Cálculo y Geometría Analítica, 1984. McGraw - Hill, México. Tercera Edición; 212-214. 